author closely evaluates other permeability factors (plasma globulins, leucotaxines and plasma kinins) and considers that they are not involved in microcirculatory regulation.

Schayer then discusses the modes of action of glucocorticoids as vasoconstrictors of the smooth muscle of the microcirculation and possible potentiators of catecholamines; thus these two actions of the glucocorticoids would combine to inhibit the effects of induced histamine on the microcirculation. The author tabulates the response of the microcirculation to various circumstances, interpreting the observed changes in terms of a balance between induced histamine synthesis on one hand and glucocorticoid status on the other.

Finally, S. B. Salvin contributes an article entitled "Immunological Aspects of the Mycoses". He reviews the fungal antigens, antibodies and serological tests in eleven mycotic diseases, while he further discusses delayed hypersensitivity, pathogenesis, resistance and immunization in these disorders. The mycoses reviewed are as follows: A.ctinomycosis, nocardiosis, aspergillosis, candidiasis, cryptococcosis, North American blastomycosis, coccidioidomycosis, histoplasmosis, chromoblastomycosis, sporotrichosis, and dermatomycosis. This is a monumental review, containing nearly 800 references to the literature, though clinical mycologists may notice the absence of data concerning Mucor, Rhizopus, and Absidia. The alarming increase in deep-seated mycotic disease that has occurred during the past ten years makes Salvin's review most timely: its value is thus two-fold, for there is a growing increase in the immunology of Fungi in general.

As can be seen from the contents, this volume richly deserves its place in the reference literature of Immunology. In an introduction, Waksman summarizes recent developments in immunology and makes a plea for the recognition of Immunopathology as a special discipline, and for the introduction of a journal devoted to this speciality. Those who eagerly await the appearance of each, now annual, addition to the Progress in Allergy series will agree that these reviews make a great contribution in themselves.

D. C. DUMONDE

\section{ANALYSIS OF PLANT DISEASE EPIDEMICS}

\section{Plant Diseases}

Epidemics and Control. By J. E. van der Plank. Pp. xvi +349. (New York: Academic Press, Inc.; London: Academic Press, Inc. (London), Ltd., 1963.) $80 \mathrm{~s}$.

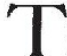
HOSE who know Dr. van der Plank or are aware of his substantial contributions to the epidemiology of plant diseases will not be led astray by the title of this book, but others may be. It is not, as one might anticipate, a description of plant disease opidemics and how to control them, but a dissertation on new methods of epidemiological analysis based on the rate at which the population of the pathogen increases (infection rate) and on the relation between the amount of inoculum and the amount of disease the inoculum produces. It also demonstrates how this kind of analysis can be applied in the control of diseases, whether by the use of resistant varieties, by sanitation or by chemicals, all of which help to reduce the amount of inoculum, or the infection rate, or both.

Plant Diseases: Epidemics and Control is intended primarily for plant pathologists and for plant breeders interested in breeding for resistance to diseases, and some of it is likely to be heavy going for those without an adequate knowledge of mathematics or who lack interest or experience in the application of mathematics to biology. The author, convinced that training in epidemiology will soon become as essential for a plant pathologist as training in mycology, virology or genetics, is clearly aware of this difficulty for he goes out of his way to help his readers. The first of the twenty-three chapters explains simply and clearly what the book is about and there is a continuous explanatory thread, in part taking the form of an introduction to each chapter, which runs through the text, encouraging and maintaining interest even through the more difficult parts. Exercises in epidemiological analysis are included at the end of each of the chapters concerned with methods, and relevant mathematical tables are provided in an appendix. The other early chapters deal with analogies drawn between rates of interest on money and disease infection rates; with methods for plotting the progress of an epidemic; and with the breakdown and analysis of infection rates to reveal how they may be influenced by various factors, such as the time newly infected tissues take to become infectious (latent period) and the time needed for symptoms to develop (incubation period), or by the effects of new host growth, gradual sterility of infected tissues and so on.

The rest of the book is mainly devoted to the illustration of how the practice of sanitation or the use of resistant varieties and fungicides may be related to the analysis of control. The effect of sanitation, very loosely defined as the process of reducing the inoculum from which an epidemic starts, is dealt with in three chapters, with special reference to potato blight, black stem rust of wheat, bunt of wheat and Fusarium wilt of tomato. Then follow seven chapters on types of disease resistance, again with potato blight and black stem rust as the cardinal examples. A clear distinction is made between vertical resistance, which is directed against some races of a pathogen but not others, and horizontal resistance (in potatoes at present called field resistance) which operates against all races. The need for and intent of this new jargon is less apparent. Vertical resistance, the usual choice of plant breeders, is preferred by the author only when there is a record of variety stability or when the disease multiplies slowly. Horizontal resistance is preferred when varietal change is frequently imposed by rapid changes in the races of the pathogen or when it is desired to improve the performance of fungicides. In biological warfare with black stem rust, for example, vertical resistance would, it is thought, leave the wheat crop wide open to destruction, whereas horizontal resistance is believed to provide an adequate defence. In chemical control of diseases, regarded here at times as a form of sanitation, the fungicidal action of the chemical is only part of the story. Successful control of an epidemic depends almost equally on weather or other environmental conditions and could be greatly promoted by better horizontal resistance in the host plants.

The text ends with two chapters on the spread, as distinct from the increase, of disease. In the second of these, the author is highly critical of many field experiments intended to provide information that can be applied to farming. In his view, experimental plots frequently do not represent accurately the farmer's fields or orchards they are meant to, because of 'representational' and 'cryptic' errors. Such errors arise, for example, when guard rows in conventional layouts are not efficient against fastmoving pathogens in reducing or preventing plot interference, and the extent of the errors may depend on other factors, including plot size and shape. Special layouts for avoiding interference and reducing representational errors are suggested. More than 200 literature references are listed, most of them to work published in the past decade.

This extremely well-written book can be of absorbing interest, even to one who finds much of the mathematics beyond him. It breaks new ground with new ideas and will without doubt lead to much discussion and argument among plant pathologists and plant breeders as well as in a wider field, for much of it will surely find a place in future training courses. 